\title{
A FINITE ELEMENT DISCRETIZATION OF THE CONTACT BETWEEN TWO MEMBRANES
}

\author{
Faker Ben Belgacem ${ }^{1}$, Christine Bernardi ${ }^{2}$, Adel Blouza ${ }^{3}$ \\ AND MarTin VOHRALÍK ${ }^{2}$
}

\begin{abstract}
From the fundamental laws of elasticity, we write a model for the contact between two membranes and we perform the analysis of the corresponding system of variational inequalities. We propose a finite element discretization of this problem and prove its well-posedness. We also establish a priori and a posteriori error estimates.
\end{abstract}

Mathematics Subject Classification. 65N30, 73K10, $73 \mathrm{~T} 05$.

Received January 18, 2008. Revised May 25, 2008.

Published online October 16, 2008.

\section{Introduction}

We are interested in the numerical simulation of the contact between two elastic membranes. We first write the model for this problem which is based on the fundamental laws of elasticity. The contact is taken into account according to the following principles:

(i) The two membranes cannot interpenetrate;

(ii) Where they are in contact, owing to Newton's action-reaction law, each membrane has an equal action on the other.

Starting from these ideas, we obtain a system made of a partial differential equation and an inequality, which seems an acceptable model for the contact from a mechanical point of view. We thus state an equivalent variational formulation which is of mixed type: The three unknowns are the position of each membrane and the action of each membrane on the other one. This kind of system appears in a large number of problems in elasticity, such as the obstacle or Signorini problems, see [5,13,18] among others. It also fits the abstract framework proposed in [2]. Relying on the results of [5,18], we prove the well-posedness of our problem.

In view of the discretization, we note that the action is sought for in a space of distributions with a nonlocal norm; this seems hardly compatible with a finite element discretization since the main advantages of such

\footnotetext{
Keywords and phrases. Unilateral contact, variational inequalities, finite elements, a priori and a posteriori analysis.

1 L.M.A.C. (E.A. 2222), Département de Génie Informatique, Université de Technologie de Compiègne, Centre de Recherches de Royallieu, B.P. 20529, 60205 Compiègne Cedex, France. faker.ben-belgacem@utc.fr

${ }^{2}$ Laboratoire Jacques-Louis Lions, C.N.R.S. \& Université Pierre et Marie Curie, B.C. 187, 4 place Jussieu, 75252 Paris Cedex 05, France. bernardi@ann.jussieu.fr; vohralik@ann.jussieu.fr

3 Laboratoire de Mathématiques Raphaël Salem (U.M.R. 6085 C.N.R.S.), Université de Rouen, avenue de l'Université, B.P. 12, 76801 Saint-Étienne-du-Rouvray, France. Adel.Blouza@univ-rouen.fr
} 
methods are their local approximation properties. Our idea is then to introduce a modified unknown by using the Riesz isomorphism: Even if it has no obvious physical meaning, it is easy to deduce from it the action of the membranes. We thus propose a standard finite element discretization of this new formulation constructed by the Galerkin method with Lagrange finite elements. We prove that the discrete problem has a unique solution and derive optimal a priori error estimates.

After the pioneering paper [1] by Ainsworth et al., a substantial work has been performed on the a posteriori analysis of variational inequalities, see, e.g., $[6,16,20]$ and the references therein. We follow the approach of Hild and Nicaise [14] since they also consider a mixed problem coupling a variational equality and an inequality. We introduce two families of error indicators: The first family concerns the residual of the variational equation (see [19], Sect. 1.2, for the basic arguments leading to the introduction of this indicator); the second family deals with the inequality and mainly represents the lack of positivity of the approximate action. We prove a posteriori error estimates which are not fully optimal, however the same lack of optimality is already observed in [14] for a similar problem. Moreover, since the upper bounds for the indicators are local, we think that they are an efficient tool for mesh adaptivity.

An outline of the paper is as follows.

- In Section 2, we explain the main arguments for the derivation of the model.

- Section 3 is devoted to the analysis of the corresponding system.

- In Section 4, we propose and study a modified variational formulation of the same problem.

- In Section 5, we describe the discrete problem and check its well-posedness.

- A priori and a posteriori error estimates for this discretization are established in Sections 6 and 7 , respectively.

- Some conclusions are given in Section 8.

\section{Derivation of the Model}

An elastic membrane is characterized by its displacement $u$ with respect to its natural configuration which is a two-dimensional domain $\omega$. The equilibrium position of the membrane, under the action of a vertical force $\mathcal{F}$, minimizes the potential energy functional

$$
J: \quad v \mapsto J(v)=\frac{1}{2} \int_{\omega} \mu(\boldsymbol{x})|\nabla v(\boldsymbol{x})|^{2} \mathrm{~d} \boldsymbol{x}-\int_{\omega} \mathcal{F}(\boldsymbol{x}) v(\boldsymbol{x}) \mathrm{d} \boldsymbol{x},
$$

where $\mu$ represents the tension of the membrane. Assuming moreover that it is fixed on its boundary, the minimization problem reads: Find $u$ in $H_{0}^{1}(\omega)$ such that

$$
\forall v \in H_{0}^{1}(\omega), \quad J(u) \leq J(v)
$$

or equivalently

$$
\begin{cases}-\operatorname{div}(\mu \operatorname{grad} u)=\mathcal{F} & \text { in } \omega \\ u=0 & \text { on } \partial \omega .\end{cases}
$$

More details can be found in [7], Chapter I, Section 1.2, for instance.

Let us now consider two elastic membranes: The first one is fixed on $\partial \omega$ at the height $g$, where $g$ is a nonnegative function, and the second one is fixed at zero. The corresponding system of equations reads, with obvious notation,

$$
\left\{\begin{array} { l l } 
{ - \operatorname { d i v } ( \mu _ { 1 } \operatorname { g r a d } u _ { 1 } ) = \mathcal { F } _ { 1 } } & { \text { in } \omega , } \\
{ u _ { 1 } = g } & { \text { on } \partial \omega , }
\end{array} \quad \left\{\begin{array}{ll}
-\operatorname{div}\left(\mu_{2} \operatorname{grad} u_{2}\right)=\mathcal{F}_{2} & \text { in } \omega \\
u_{2}=0 & \text { on } \partial \omega .
\end{array}\right.\right.
$$

We are interested in the case where the membranes interact. Therefore, if $\lambda$ represents the action of the second membrane on the first one (equivalently, $-\lambda$ represents the action of the first membrane on the second one), 
we have

$$
\mathcal{F}_{1}=f_{1}+\lambda, \quad \mathcal{F}_{2}=f_{2}-\lambda,
$$

where the $f_{i}$ are external forces.

It follows from the definition of $\lambda$ that

$$
\lambda \geq 0 \quad \text { in } \omega .
$$

Moreover, clearly the two membranes cannot interpenetrate: This yields the condition

$$
u_{1}-u_{2} \geq 0 \quad \text { in } \omega \text {. }
$$

Finally, we note that, where the membranes are not in contact, i.e., where $u_{1}-u_{2}>0$, the interaction $\lambda$ vanishes. This leads to the equation

$$
\left(u_{1}-u_{2}\right) \lambda=0 \quad \text { in } \omega .
$$

Remark 2.1. The previous equations constitute a mixed formulation of the mechanical problem with three unknowns: the displacements $u_{1}$ and $u_{2}$, and the action-reaction $\lambda$, which can be considered as a Lagrange multiplier. A simpler form of these equations consists in minimizing the functional

$$
\mathcal{J}: \quad\left(v_{1}, v_{2}\right) \mapsto \mathcal{J}\left(v_{1}, v_{2}\right)=\sum_{i=1}^{2}\left(\frac{1}{2} \int_{\omega} \mu_{i}(\boldsymbol{x})\left|\nabla v_{i}(\boldsymbol{x})\right|^{2} \mathrm{~d} \boldsymbol{x}-\int_{\omega} f_{i}(\boldsymbol{x}) v_{i}(\boldsymbol{x}) \mathrm{d} \boldsymbol{x}\right)
$$

on the convex set made by the pairs $\left(v_{1}, v_{2}\right)$ such that $v_{1}-v_{2}$ is nonnegative on $\omega$. The links between these two problems are brought to light in the next section.

Remark 2.2. It can be noted that, in the case without contact, i.e., $u_{1}-u_{2}>0$, system (2.3) becomes

$$
\begin{cases}-\operatorname{div}\left(\mu_{1} \operatorname{grad} u_{1}\right)=f_{1} & \text { in } \omega, \\ -\operatorname{div}\left(\mu_{2} \operatorname{grad} u_{2}\right)=f_{2} & \text { in } \omega, \\ u_{1}=g & \text { on } \partial \omega, \\ u_{2}=0 & \text { on } \partial \omega,\end{cases}
$$

while in the case $u_{1}=u_{2}=u$ of full contact (this implies $g=0$ ), it becomes

$$
\begin{cases}-\operatorname{div}\left(\left(\mu_{1}+\mu_{2}\right) \operatorname{grad} u\right)=f_{1}+f_{2} & \text { in } \omega \\ u=0 & \text { on } \partial \omega .\end{cases}
$$

So inequalities (2.5) and (2.6) are really linked to the zone of contact between the membranes.

We are now interested in the analysis of the system (2.3) to (2.7). In what follows, only for simplicity, we take the boundary condition $g$ equal to zero and assume that the coefficients $\mu_{i}$ are positive constants. 


\section{Analysis of the COntinuous problem}

Let $\omega$ be a bounded open set in $\mathbb{R}^{2}$, with a Lipschitz-continuous boundary. In view of the previous section, we are led to consider the following system

$$
\begin{cases}-\mu_{1} \Delta u_{1}-\lambda=f_{1} & \text { in } \omega, \\ -\mu_{2} \Delta u_{2}+\lambda=f_{2} & \text { in } \omega, \\ u_{1}-u_{2} \geq 0, \quad \lambda \geq 0, & \left(u_{1}-u_{2}\right) \lambda=0 \\ u_{1}=0 & \text { in } \omega, \\ u_{2}=0 & \text { on } \partial \omega\end{cases}
$$

where the coefficients $\mu_{1}$ and $\mu_{2}$ are positive constants. The unknowns are the displacements $u_{1}$ and $u_{2}$ of the two membranes, and the Lagrange multiplier $\lambda$.

We now intend to write a variational formulation of system (3.1). In order to do this, we consider the full scale of Sobolev spaces $H^{s}(\omega), s \geq 0$, equipped with the usual norms (and semi-norms when $s$ is a positive integer). We also need the space $H_{0}^{1}(\omega)$ of functions in $H^{1}(\omega)$ which vanish on $\partial \omega$, and we denote by $H^{-1}(\omega)$ its dual space. Next, we introduce the convex subset

$$
\Lambda=\left\{v \in H_{0}^{1}(\omega) ; v \geq 0 \text { a.e. in } \omega\right\}
$$

and finally the convex subset $\Lambda^{*}$ of distributions $\chi$ in $H^{-1}(\omega)$ such that

$$
\forall v \in \Lambda, \quad\langle\chi, v\rangle \geq 0
$$

where from now on $\langle\cdot, \cdot\rangle$ stands for the duality pairing between $H^{-1}(\omega)$ and $H_{0}^{1}(\omega)$.

So we consider the following variational problem, for any data $\left(f_{1}, f_{2}\right)$ in $H^{-1}(\omega) \times H^{-1}(\omega)$ :

Find $\left(u_{1}, u_{2}, \lambda\right)$ in $H_{0}^{1}(\omega) \times H_{0}^{1}(\omega) \times \Lambda^{*}$ such that

$$
\begin{aligned}
& \forall\left(v_{1}, v_{2}\right) \in H_{0}^{1}(\omega) \times H_{0}^{1}(\omega), \quad \sum_{i=1}^{2} \mu_{i} \int_{\omega}\left(\operatorname{grad} u_{i}\right)(\boldsymbol{x}) \cdot\left(\operatorname{grad} v_{i}\right)(\boldsymbol{x}) \mathrm{d} \boldsymbol{x} \\
& \forall \chi \in \Lambda^{*}, \quad\left\langle\chi-\lambda, u_{1}-u_{2}\right\rangle \geq 0 .
\end{aligned}
$$

We must now check the equivalence of this problem with system (3.1).

Proposition 3.1. Problems (3.1) and (3.4) are equivalent, in the sense that any triple $\left(u_{1}, u_{2}, \lambda\right)$ in $H^{1}(\omega) \times$ $H^{1}(\omega) \times H^{-1}(\omega)$ is a solution of (3.1) if and only if it is a solution of (3.4).

Proof. Since the fourth and fifth lines in (3.1) are obviously equivalent to the fact that $u_{1}$ and $u_{2}$ belong to $H_{0}^{1}(\omega)$, we now verify the equivalence of the other lines.

(1) Let $\mathscr{D}(\omega)$ be the space of infinitely differentiable functions with a compact support in $\omega$. Multiplying the first line of $(3.1)$ by a function $v_{1}$ in $\mathscr{D}(\omega)$ and the second line by a function $v_{2}$ in $\mathscr{D}(\omega)$, summing these two equations and integrating by parts yield that the first line of $(3.4)$ is satisfied for all pairs $\left(v_{1}, v_{2}\right)$ in $\mathscr{D}(\omega)^{2}$. Thus, it follows 
from the density of $\mathscr{D}(\omega)$ in $H_{0}^{1}(\omega)$ that this line is satisfied for all $\left(v_{1}, v_{2}\right)$ in $H_{0}^{1}(\omega)^{2}$. Conversely, by letting $v_{1}$ run through $\mathscr{D}(\omega)$ and taking $v_{2}$ equal to zero, next by taking $v_{1}$ equal to zero and letting $v_{2}$ run through $\mathscr{D}(\omega)$, we observe that the first line of (3.4) implies the first two lines of (3.1) in the sense of distributions.

(2) Let $\left(u_{1}, u_{2}, \lambda\right)$ satisfy the third line of (3.1). Thus, $\lambda$ belongs to $\Lambda^{*}$ and it follows from the definition of $\Lambda^{*}$ that, for all $\chi$ in $\Lambda^{*}$,

$$
\left\langle\chi-\lambda, u_{1}-u_{2}\right\rangle=\left\langle\chi, u_{1}-u_{2}\right\rangle \geq 0 .
$$

Conversely, if $\lambda$ belongs to $\Lambda^{*}$ and $\left(u_{1}, u_{2}, \lambda\right)$ satisfies the second line of (3.4), then $\lambda$ is nonnegative. Moreover, taking $\chi$ equal to the sum of $\lambda$ and of the characteristic function $\chi_{\mathcal{O}}$ of any measurable subset $\mathcal{O}$ of $\omega$ (this $\chi_{\mathcal{O}}$ obviously belongs to $\Lambda^{*}$ ) yields that

$$
\int_{\mathcal{O}}\left(u_{1}-u_{2}\right)(\boldsymbol{x}) \mathrm{d} \boldsymbol{x} \geq 0
$$

whence the nonnegativity of $u_{1}-u_{2}$. Finally, taking $\chi$ equal to zero yields that

$$
\left\langle\lambda, u_{1}-u_{2}\right\rangle \leq 0
$$

and combining this with the previous properties gives the equality $\left(u_{1}-u_{2}\right) \lambda=0$.

Setting $\boldsymbol{u}=\left(u_{1}, u_{2}\right)$ and $\boldsymbol{v}=\left(v_{1}, v_{2}\right)$, we consider the bilinear form defined by

$$
a(\boldsymbol{u}, \boldsymbol{v})=\sum_{i=1}^{2} \mu_{i} \int_{\omega}\left(\operatorname{grad} u_{i}\right)(\boldsymbol{x}) \cdot\left(\operatorname{grad} v_{i}\right)(\boldsymbol{x}) \mathrm{d} \boldsymbol{x} .
$$

Its continuity on $H^{1}(\omega)^{2} \times H^{1}(\omega)^{2}$ is obvious and its ellipticity on $H_{0}^{1}(\omega)^{2}$ follows from a Poincaré-Friedrichs inequality: There exists a constant $\alpha>0$ only depending on $\omega$ and on the $\mu_{i}$ such that

$$
\forall \boldsymbol{v} \in H_{0}^{1}(\omega)^{2}, \quad a(\boldsymbol{v}, \boldsymbol{v}) \geq \alpha\|\boldsymbol{v}\|_{H^{1}(\omega)^{2}}^{2} .
$$

With the same notation, we also introduce the bilinear form

$$
b(\boldsymbol{v}, \chi)=-\left\langle\chi, v_{1}-v_{2}\right\rangle,
$$

which is continuous on $H_{0}^{1}(\omega)^{2} \times H^{-1}(\omega)$ owing to the definition of $H^{-1}(\omega)$. The inf-sup condition that we now state is also a direct consequence of the Riesz theorem. However we prefer to give the proof for completeness.

Lemma 3.2. There exists a constant $\beta>0$ such that

$$
\forall \chi \in H^{-1}(\omega), \quad \sup _{\boldsymbol{v} \in H_{0}^{1}(\omega)^{2}} \frac{b(\boldsymbol{v}, \chi)}{\|\boldsymbol{v}\|_{H^{1}(\omega)^{2}}} \geq \beta\|\chi\|_{H^{-1}(\omega)} .
$$

Proof. For any $\chi$ in $H^{-1}(\omega)$, it follows from the Lax-Milgram lemma that the problem:

Find $w$ in $H_{0}^{1}(\omega)$ such that

$$
\forall v \in H_{0}^{1}(\omega), \quad \int_{\omega}(\operatorname{grad} w)(\boldsymbol{x}) \cdot(\operatorname{grad} v)(\boldsymbol{x}) \mathrm{d} \boldsymbol{x}=\langle\chi, v\rangle
$$

has a unique solution. Moreover, owing to the definition of the norm of $H^{-1}(\omega)$ as a dual norm, we have

$$
\|\chi\|_{H^{-1}(\omega)} \leq|w|_{H^{1}(\omega)} .
$$


On the other hand, taking $\boldsymbol{v}=(0, w)$ yields

$$
b(\boldsymbol{v}, \chi)=|w|_{H^{1}(\omega)}^{2} .
$$

So the desired result follows from the two previous lines, combined with a Poincaré-Friedrichs inequality.

Problem (3.4) fits the abstract framework introduced in [18], so that its well-posedness can be derived from [18], Theorem 2.3. The existence and uniqueness results also follow from [2], Theorem 2.4.2. However, we prefer to give a direct proof of this result, in view of its analogue for the discrete problem. We begin by proving an upper bound for the norm of the solution.

Lemma 3.3. Any solution $\left(u_{1}, u_{2}, \lambda\right)$ of problem (3.4) satisfies

$$
\left\|u_{1}\right\|_{H^{1}(\omega)}+\left\|u_{2}\right\|_{H^{1}(\omega)}+\|\lambda\|_{H^{-1}(\omega)} \leq c\left(\left\|f_{1}\right\|_{H^{-1}(\omega)}+\left\|f_{2}\right\|_{H^{-1}(\omega)}\right) .
$$

Proof. We first observe from the second line of (3.4) that taking $\chi$ equal to 0 gives

$$
\left\langle\lambda, u_{1}-u_{2}\right\rangle \leq 0 .
$$

Thus, taking $v_{1}$ equal to $u_{1}$ and $v_{2}$ equal to $u_{2}$ in the first line of (3.4), we derive thanks to Cauchy-Schwarz inequalities

$$
\min \left\{\mu_{1}, \mu_{2}\right\}\left(\left|u_{1}\right|_{H^{1}(\omega)}+\left|u_{2}\right|_{H^{1}(\omega)}\right) \leq \sqrt{2}\left(\left\|f_{1}\right\|_{H^{-1}(\omega)}+\left\|f_{2}\right\|_{H^{-1}(\omega)}\right) .
$$

On the other hand, applying the inf-sup condition (3.8) and using the first line of (3.4) to evaluate $b(\boldsymbol{v}, \lambda)$ yield

$$
\|\lambda\|_{H^{-1}(\omega)} \leq c\left(\left|u_{1}\right|_{H^{1}(\omega)}+\left|u_{2}\right|_{H^{1}(\omega)}+\left\|f_{1}\right\|_{H^{-1}(\omega)}+\left\|f_{2}\right\|_{H^{-1}(\omega)}\right) .
$$

So (3.9) follows from the two previous estimates combined with the Poincaré-Friedrichs inequality.

To go further, we introduce the new convex set

$$
\mathcal{K}=\left\{\left(v_{1}, v_{2}\right) \in H_{0}^{1}(\omega) \times H_{0}^{1}(\omega) ; v_{1}-v_{2} \geq 0 \text { a.e. in } \omega\right\}
$$

and we consider the reduced problem:

Find $\left(u_{1}, u_{2}\right)$ in $\mathcal{K}$ such that

$$
\forall\left(v_{1}, v_{2}\right) \in \mathcal{K}, \quad \sum_{i=1}^{2} \mu_{i} \int_{\omega}\left(\operatorname{grad} u_{i}\right)(\boldsymbol{x}) \cdot\left(\operatorname{grad}\left(v_{i}-u_{i}\right)\right)(\boldsymbol{x}) \mathrm{d} \boldsymbol{x} \geq \sum_{i=1}^{2}\left\langle f_{i}, v_{i}-u_{i}\right\rangle .
$$

The reason for this is stated in the next lemma.

Lemma 3.4. For any solution $\left(u_{1}, u_{2}, \lambda\right)$ of problem (3.4), the pair $\left(u_{1}, u_{2}\right)$ is a solution of problem (3.11).

Proof. Let $\left(u_{1}, u_{2}, \lambda\right)$ be a solution of problem (3.4). Owing to Proposition 3.1, it satisfies the third line of (3.1), so that $\left(u_{1}, u_{2}\right)$ belongs to $\mathcal{K}$. On the other hand, for any $\left(v_{1}, v_{2}\right)$ in $\mathcal{K}$, it follows from the definitions of $\Lambda^{*}$ and $\mathcal{K}$ that

The second line of (3.4) with $\chi=0$ also yields that

$$
-\left\langle\lambda, v_{1}-v_{2}\right\rangle \leq 0 .
$$

$$
\left\langle\lambda, u_{1}-u_{2}\right\rangle \leq 0 .
$$

Replacing each $v_{i}$ by $v_{i}-u_{i}$ in the first line of (3.4) and inserting these last two inequalities yields (3.11).

We are now in a position to state the main result of this section. 
Theorem 3.5. For any data $\left(f_{1}, f_{2}\right)$ in $H^{-1}(\omega) \times H^{-1}(\omega)$, problem $(3.4)$ has a unique solution $\left(u_{1}, u_{2}, \lambda\right)$ in $H_{0}^{1}(\omega) \times H_{0}^{1}(\omega) \times \Lambda^{*}$. Moreover, this solution satisfies estimate (3.9).

Proof. Since estimate (3.9) is established in Lemma 3.3, we prove successively the existence and the uniqueness of the solution.

(1) Owing to the ellipticity property (3.6), the existence of a solution $\left(u_{1}, u_{2}\right)$ of problem (3.11) is a direct consequence of the Lions-Stampacchia theorem [15], see [13], Theorem 3.1, for instance. We set

$$
\mathcal{L}(\boldsymbol{v})=a(\boldsymbol{u}, \boldsymbol{v})-\sum_{i=1}^{2}\left\langle f_{i}, v_{i}\right\rangle,
$$

and observe from (3.11) by taking $\boldsymbol{v}$ equal to $\mathbf{0}$, next to $2 \boldsymbol{u}$, that

$$
\mathcal{L}(\boldsymbol{u})=0 .
$$

On the other hand, the kernel $V$ of the form $b(\cdot, \cdot)$ is characterized by

$$
V=\left\{\left(v_{1}, v_{2}\right) \in H_{0}^{1}(\omega) \times H_{0}^{1}(\omega) ; v_{1}-v_{2}=0 \text { a.e. in } \omega\right\} .
$$

Let $\boldsymbol{v}=\left(v_{1}, v_{2}\right)$ be any pair in $V$. Thus, it is readily checked that both $\boldsymbol{v}$ and $-\boldsymbol{v}$ belong to $\mathcal{K}$. It follows from (3.11) and (3.13) that $\mathcal{L}(\boldsymbol{v})$ is nonnegative and from (3.11) with $\boldsymbol{v}$ replaced by $-\boldsymbol{v}$ and (3.13) that $\mathcal{L}(\boldsymbol{v})$ is nonpositive. So, $\mathcal{L}$ vanishes on $V$. Therefore, thanks to the inf-sup condition (3.8), there exists (see [11], Chap. I, Lem. 4.1, for instance) a $\lambda$ in $H^{-1}(\omega)$ such that

$$
\forall \boldsymbol{v} \in H_{0}^{1}(\omega)^{2}, \quad b(\boldsymbol{v}, \lambda)=\mathcal{L}(\boldsymbol{v}) .
$$

So, the triple $\left(u_{1}, u_{2}, \lambda\right)$ satisfies the first line of (3.4), and $\lambda$ belongs to $\Lambda^{*}$ owing to problem (3.11). It also follows from the definition of $\Lambda^{*}$ and (3.13) that

$$
\forall \chi \in \Lambda^{*}, \quad\left\langle\chi-\lambda, u_{1}-u_{2}\right\rangle=\left\langle\chi, u_{1}-u_{2}\right\rangle \geq 0,
$$

which is the second line of (3.4). So, we have established the existence result.

(2) Let $\left(u_{1}, u_{2}, \lambda\right)$ and $\left(\tilde{u}_{1}, \tilde{u}_{2}, \tilde{\lambda}\right)$ be two solutions of problem (3.4). Then, both $\left(u_{1}, u_{2}\right)$ and $\left(\tilde{u}_{1}, \tilde{u}_{2}\right)$ are solutions of (3.11). Combining the Lions-Stampacchia theorem with (3.6) yields that $u_{1}=\tilde{u}_{1}$ and $u_{2}=\tilde{u}_{2}$. We then derive from the first line of (3.4) that

$$
\forall\left(v_{1}, v_{2}\right) \in H_{0}^{1}(\omega) \times H_{0}^{1}(\omega), \quad\left\langle\lambda, v_{1}-v_{2}\right\rangle=\left\langle\tilde{\lambda}, v_{1}-v_{2}\right\rangle,
$$

whence $\lambda=\tilde{\lambda}$. This leads to the uniqueness result.

We conclude this section with a regularity result. The arguments are the same as in [4] but simpler, so that we prefer to give a direct proof.

Proposition 3.6. For any data $\left(f_{1}, f_{2}\right)$ in $L^{2}(\omega) \times L^{2}(\omega)$, the solution $\left(u_{1}, u_{2}, \lambda\right)$ of problem (3.4) belongs to $H^{s+1}(\omega) \times H^{s+1}(\omega) \times L^{2}(\omega)$, with

(i) $s=\frac{1}{2}$ in the general case;

(ii) $s=1$ when $\omega$ is convex or of class $\mathscr{C}^{1,1}$;

(iii) $s<\frac{\pi}{\alpha}$ when $\omega$ is a polygon with its largest angle equal to $\alpha$.

Moreover, for any open set $\omega^{*}$ such that $\bar{\omega}^{*} \subset \omega$, the restriction of the solution $\left(u_{1}, u_{2}, \lambda\right)$ of problem (3.4) to $\omega^{*}$ belongs to $H^{2}\left(\omega^{*}\right) \times H^{2}\left(\omega^{*}\right) \times L^{2}\left(\omega^{*}\right)$. 
Proof. For any $\varepsilon>0$ and for $i=1,2$, the problem:

Find $u_{\varepsilon i}$ in $H_{0}^{1}(\omega)$ such that

$$
u_{\varepsilon i}-\varepsilon \Delta u_{\varepsilon i}=u_{i}
$$

has a unique solution. Moreover, it follows from the maximum principle and the fact that $\left(u_{1}, u_{2}\right)$ belongs to $\mathcal{K}$ that $u_{\varepsilon 1} \geq u_{\varepsilon 2}$, so that $\left(u_{\varepsilon 1}, u_{\varepsilon 2}\right)$ also belongs to $\mathcal{K}$. Thus, when taking $v_{i}$ equal to $u_{\varepsilon i}$ in problem (3.11), we observe that

$$
\sum_{i=1}^{2} \mu_{i} \int_{\omega}\left(\operatorname{grad} u_{i}\right)(\boldsymbol{x}) \cdot\left(\operatorname{grad}\left(u_{i}-u_{\varepsilon i}\right)\right)(\boldsymbol{x}) \mathrm{d} \boldsymbol{x} \leq \sum_{i=1}^{2}\left\langle f_{i}, u_{i}-u_{\varepsilon i}\right\rangle
$$

or equivalently

$$
\sum_{i=1}^{2} \mu_{i}\left(\left|u_{i}-u_{\varepsilon i}\right|_{H^{1}(\omega)}^{2}+\int_{\omega}\left(\operatorname{grad} u_{\varepsilon i}\right)(\boldsymbol{x}) \cdot\left(\operatorname{grad}\left(u_{i}-u_{\varepsilon i}\right)\right)(\boldsymbol{x}) \mathrm{d} \boldsymbol{x}\right) \leq \sum_{i=1}^{2}\left\langle f_{i}, u_{i}-u_{\varepsilon i}\right\rangle .
$$

It thus follows from the variational formulation of problem (3.15) that

$$
\varepsilon^{-1} \sum_{i=1}^{2} \mu_{i}\left\|u_{i}-u_{\varepsilon i}\right\|_{L^{2}(\omega)} \leq c \sum_{i=1}^{2}\left\|f_{i}\right\|_{L^{2}(\omega)}
$$

So, the $u_{\varepsilon i}$ tend to $u_{i}$ strongly in $L^{2}(\omega)$ when $\varepsilon$ tends to zero. On the other hand, it follows from the previous estimate that

$$
\sum_{i=1}^{2} \mu_{i}\left\|\Delta u_{\varepsilon i}\right\|_{L^{2}(\omega)} \leq c \sum_{i=1}^{2}\left\|f_{i}\right\|_{L^{2}(\omega)}
$$

Therefore, there exists a subsequence of the $u_{\varepsilon i}$ such that $\Delta u_{\varepsilon i}$ converges weakly in $L^{2}(\omega)$. Since its limit is necessarily $\Delta u_{i}$, each $\Delta u_{i}$ belongs to $L^{2}(\omega)$. We conclude in two steps:

(1) Since each $u_{i}$ belongs to $H_{0}^{1}(\omega)$ and is such that $\Delta u_{i}$ belongs to $L^{2}(\omega)$, we derive from [12], Theorem 3.2.1.2, and [12], Section 4.3, for instance that $u_{i}$ belongs to $H^{s+1}(\omega)$ for the values of $s$ indicated in the proposition and also to $H^{2}\left(\omega^{*}\right)$.

(2) Finally, $\lambda=-\mu_{1} \Delta u_{1}-f_{1}$ belongs to $L^{2}(\omega)$.

Remark 3.7. Assume now that the $\mu_{i}, i=1$ or 2 , are bounded functions satisfying for some positive constants $\mu_{\mathrm{b}}$ and $\mu_{\sharp}$

$$
\text { for a.e. } \boldsymbol{x} \text { in } \omega, \quad \mu_{b} \leq \mu_{i}(\boldsymbol{x}) \leq \mu_{\sharp} \text {. }
$$

Thus, replacing the form $a(\cdot, \cdot)$ defined in $(3.5)$ by

$$
a(\boldsymbol{u}, \boldsymbol{v})=\sum_{i=1}^{2} \int_{\omega} \mu_{i}(\boldsymbol{x})\left(\operatorname{grad} u_{i}\right)(\boldsymbol{x}) \cdot\left(\operatorname{grad} v_{i}\right)(\boldsymbol{x}) \mathrm{d} \boldsymbol{x},
$$

and using the same arguments as previously yield that Proposition 3.1 and Theorem 3.5 still hold in this case. The regularity results seem however weaker than those stated in Proposition 3.6.

Remark 3.8. Let us have a look at the more realistic case where the boundary condition on $u_{1}$ is replaced by

$$
u_{1}=g \quad \text { on } \partial \omega
$$


where $g$ is a nonnegative function in $H^{\frac{1}{2}}(\partial \omega)$. Using the harmonic lifting $\bar{g}$ of $g$ (which is nonnegative on $\omega$ ) and setting $u_{1}^{*}=u_{1}-g$, we observe that $\left(u_{1}^{*}, u_{2}, \lambda\right)$ is now a solution in $H_{0}^{1}(\omega) \times H_{0}^{1}(\omega) \times \Lambda^{*}$ of the problem

$$
\begin{aligned}
& \forall\left(v_{1}, v_{2}\right) \in H_{0}^{1}(\omega) \times H_{0}^{1}(\omega), \\
& \mu_{1} \int_{\omega}\left(\operatorname{grad} u_{1}^{*}\right)(\boldsymbol{x}) \cdot\left(\operatorname{grad} v_{1}\right)(\boldsymbol{x}) \mathrm{d} \boldsymbol{x}+\mu_{2} \int_{\omega}\left(\operatorname{grad} u_{2}\right)(\boldsymbol{x}) \cdot\left(\operatorname{grad} v_{2}\right)(\boldsymbol{x}) \mathrm{d} \boldsymbol{x} \\
& \forall \chi \in \Lambda^{*}, \quad\left\langle\chi-\lambda, u_{1}^{*}-u_{2}\right\rangle \geq-\langle\chi-\lambda, \bar{g}\rangle .
\end{aligned}
$$

Even if this system still fits the abstract framework of [5], the definition of $\Lambda^{*}$ must be modified in order to give a sense to the right-hand side of the last line. So, the study of this problem requires a new formulation where the unknown $\lambda$ is sought for in $L^{2}(\omega)$ and is under our consideration.

\section{Another variational formulation}

Problem (3.4) does not seem appropriate for a finite element discretization: Indeed, the unknown $\lambda$ is sought for in the space $H^{-1}(\omega)$ and the norm of this space is not local in the sense that it cannot be written as the sum of local norms, which seems in contradiction with the local approximation properties of the finite element spaces. So the idea of this section is to give up the physical unknown $\lambda$ and replace it by a nonphysical one which now belongs to $H_{0}^{1}(\omega)$.

This approach relies on the use of the Riesz operator $\mathcal{R}$ which, with any distribution $\chi$ in $H^{-1}(\omega)$, associates the solution $\varphi$ in $H^{1}(\omega)$ of the problem

$$
\begin{cases}-\Delta \varphi=\chi & \text { in } \omega, \\ \varphi=0 & \text { on } \partial \omega .\end{cases}
$$

Indeed, this operator is an isomorphism from $H^{-1}(\omega)$ onto $H_{0}^{1}(\omega)$ and even an isometry when $H_{0}^{1}(\omega)$ is equipped with the norm $|\cdot|_{H^{1}(\omega)}$.

Let us introduce the new cone

$$
\widetilde{\Lambda}^{*}=\left\{\varphi \in H_{0}^{1}(\omega) ; \forall v \in \Lambda, \int_{\omega}(\operatorname{grad} \varphi)(\boldsymbol{x}) \cdot(\operatorname{grad} v)(\boldsymbol{x}) \mathrm{d} \boldsymbol{x} \geq 0\right\} .
$$

It can be checked that $\widetilde{\Lambda}^{*}$ is imbedded in $\Lambda$ but simple one-dimensional counter-examples prove that the converse imbedding is false. For any data $\left(f_{1}, f_{2}\right)$ in $H^{-1}(\omega) \times H^{-1}(\omega)$, we now consider the problem:

Find $\left(u_{1}, u_{2}, \sigma\right)$ in $H_{0}^{1}(\omega) \times H_{0}^{1}(\omega) \times \widetilde{\Lambda}^{*}$ such that

$$
\begin{aligned}
& \forall\left(v_{1}, v_{2}\right) \in H_{0}^{1}(\omega) \times H_{0}^{1}(\omega), \\
& \sum_{i=1}^{2} \mu_{i} \int_{\omega}\left(\operatorname{grad} u_{i}\right)(\boldsymbol{x}) \cdot\left(\operatorname{grad} v_{i}\right)(\boldsymbol{x}) \mathrm{d} \boldsymbol{x} \\
& -\int_{\omega}(\operatorname{grad} \sigma)(\boldsymbol{x}) \cdot\left(\operatorname{grad}\left(v_{1}-v_{2}\right)\right)(\boldsymbol{x}) \mathrm{d} \boldsymbol{x}=\sum_{i=1}^{2}\left\langle f_{i}, v_{i}\right\rangle, \\
& \forall \varphi \in \widetilde{\Lambda}^{*}, \quad \int_{\omega}(\operatorname{grad}(\varphi-\sigma))(\boldsymbol{x}) \cdot\left(\operatorname{grad}\left(u_{1}-u_{2}\right)\right)(\boldsymbol{x}) \mathrm{d} \boldsymbol{x} \geq 0 .
\end{aligned}
$$

The equivalence of problems (3.4) and (4.3) follows from the definition of the operator $\mathcal{R}$. 
Proposition 4.1. Problems (3.4) and (4.3) are equivalent, in the sense that:

(i) If a triple $\left(u_{1}, u_{2}, \lambda\right)$ is a solution of (3.4), the triple $\left(u_{1}, u_{2}, \mathcal{R} \lambda\right)$ is a solution of problem (4.3);

(ii) If a triple $\left(u_{1}, u_{2}, \sigma\right)$ is a solution of (4.3), the triple $\left(u_{1}, u_{2},-\Delta \sigma\right)$ is a solution of problem (3.4).

Problem (4.3) again fits the frameworks of $[2,18]$. Moreover, the bilinear form defined by

$$
\tilde{b}(\boldsymbol{v}, \varphi)=-\int_{\omega}(\operatorname{grad} \varphi)(\boldsymbol{x}) \cdot\left(\operatorname{grad}\left(v_{1}-v_{2}\right)\right)(\boldsymbol{x}) \mathrm{d} \boldsymbol{x},
$$

still satisfies the inf-sup condition (which is easily derived by taking $\boldsymbol{v}$ equal to $(0, \varphi)$ )

$$
\forall \varphi \in H_{0}^{1}(\omega), \quad \sup _{\boldsymbol{v} \in H_{0}^{1}(\omega)^{2}} \frac{\tilde{b}(\boldsymbol{v}, \varphi)}{|\boldsymbol{v}|_{H^{1}(\omega)^{2}}} \geq|\varphi|_{H^{1}(\omega)} .
$$

But, in any case, the well-posedness of problem (4.3) is a direct consequence of Theorem 3.5 and Proposition 4.1.

Theorem 4.2. For any data $\left(f_{1}, f_{2}\right)$ in $H^{-1}(\omega) \times H^{-1}(\omega)$, problem $(4.3)$ has a unique solution $\left(u_{1}, u_{2}, \sigma\right)$ in $H_{0}^{1}(\omega) \times H_{0}^{1}(\omega) \times \widetilde{\Lambda}^{*}$. Moreover, this solution satisfies the estimate

$$
\left\|u_{1}\right\|_{H^{1}(\omega)}+\left\|u_{2}\right\|_{H^{1}(\omega)}+\|\sigma\|_{H^{1}(\omega)} \leq c\left(\left\|f_{1}\right\|_{H^{-1}(\omega)}+\left\|f_{2}\right\|_{H^{-1}(\omega)}\right) .
$$

Analogous regularity properties as stated in Proposition 3.6 still hold for problem (4.3); their proof only requires a further application of [12], Theorem 3.2.1.2 and [12], Section 4.3.

Proposition 4.3. For any data $\left(f_{1}, f_{2}\right)$ in $L^{2}(\omega) \times L^{2}(\omega)$, the solution $\left(u_{1}, u_{2}, \sigma\right)$ of problem (4.3) belongs to $H^{s+1}(\omega) \times H^{s+1}(\omega) \times H^{s+1}(\omega)$, with

(i) $s=\frac{1}{2}$ in the general case;

(ii) $s=1$ when $\omega$ is convex or of class $\mathscr{C}^{1,1}$;

(iii) $s<\frac{\pi}{\alpha}$ when $\omega$ is a polygon with its largest angle equal to $\alpha$.

Moreover, for any open set $\omega^{*}$ such that $\bar{\omega}^{*} \subset \omega$, the restriction of the solution $\left(u_{1}, u_{2}, \lambda\right)$ of problem (3.4) to $\omega^{*}$ belongs to $H^{2}\left(\omega^{*}\right) \times H^{2}\left(\omega^{*}\right) \times H^{2}\left(\omega^{*}\right)$.

The next lemma is aimed to check the consistency of the new formulation and deals with the set $\mathcal{K}$ introduced in (3.10) (see [5], Sect. 2, for the consequences of this result).

Lemma 4.4. The cone $\mathcal{K}$ defined in (3.10) coincides with the set

$$
\mathcal{K}^{*}=\left\{\boldsymbol{v}=\left(v_{1}, v_{2}\right) \in H_{0}^{1}(\omega) \times H_{0}^{1}(\omega) ; \forall \varphi \in \widetilde{\Lambda}^{*}, \tilde{b}(\boldsymbol{v}, \varphi) \leq 0\right\} .
$$

Proof. It follows from the definitions (4.2) of $\widetilde{\Lambda}^{*}$ and $(4.4)$ of $\tilde{b}(\cdot, \cdot)$ that $\mathcal{K}$ is contained in $\mathcal{K}^{*}$. Conversely, let $\boldsymbol{v}=\left(v_{1}, v_{2}\right)$ be any element of $\mathcal{K}^{*}$. Using the same arguments as in the proof of Proposition 3.1, i.e., letting $\varphi$ in the definition of $\mathcal{K}^{*}$ run through the $\mathcal{R}\left(\chi_{\mathcal{O}}\right)$, where $\chi_{\mathcal{O}}$ is the characteristic function of a measurable subset $\mathcal{O}$ of $\omega$, yields that, for any such subset,

$$
\int_{\mathcal{O}}\left(v_{1}-v_{2}\right)(\boldsymbol{x}) \mathrm{d} \boldsymbol{x} \geq 0
$$

Thus $\boldsymbol{v}$ belongs to $\mathcal{K}$.

To conclude, we note that the action of $\lambda$ on a membrane with displacement $v$ can easily be recovered from the formula

$$
\forall v \in H_{0}^{1}(\omega), \quad\langle\lambda, v\rangle=\int_{\omega}(\operatorname{grad} \sigma)(\boldsymbol{x}) \cdot(\operatorname{grad} v)(\boldsymbol{x}) \mathrm{d} \boldsymbol{x} .
$$




\section{The DISCRETE PROBLEM}

Let $\left(\mathcal{T}_{h}\right)_{h}$ be a regular family of triangulations of $\omega$ (by triangles), in the usual sense that:

- For each $h, \bar{\omega}$ is the union of all elements of $\mathcal{T}_{h}$;

- The intersection of two different elements of $\mathcal{T}_{h}$, if not empty, is a vertex or a whole edge of both of them;

- The ratio of the diameter $h_{K}$ of any element $K$ of $\mathcal{T}_{h}$ to the diameter of its inscribed circle is smaller than a constant independent of $h$.

As standard, $h$ denotes the maximum of the diameters $h_{K}, K \in \mathcal{T}_{h}$. In what follows, $c, c^{\prime}, \ldots$, stand for generic constants which may vary from line to line but are always independent of $h$.

The basic discrete space is chosen as

$$
\mathbb{X}_{h}=\left\{v_{h} \in H_{0}^{1}(\omega) ; \forall K \in \mathcal{T}_{h},\left.v_{h}\right|_{K} \in \mathcal{P}_{1}(K)\right\}
$$

where $\mathcal{P}_{1}(K)$ denotes the space of restrictions to $K$ of affine functions, i.e., of polynomials with two variables and total degree $\leq 1$. Thus, in analogy with the previous sections, we introduce the discrete cones

$$
\Lambda_{h}=\left\{v_{h} \in \mathbb{X}_{h} ; v_{h} \geq 0 \text { in } \omega\right\}
$$

and

$$
\widetilde{\Lambda}_{h}^{*}=\left\{\varphi_{h} \in \mathbb{X}_{h} ; \forall v_{h} \in \Lambda_{h}, \int_{\omega}\left(\operatorname{grad} \varphi_{h}\right)(\boldsymbol{x}) \cdot\left(\operatorname{grad} v_{h}\right)(\boldsymbol{x}) \mathrm{d} \boldsymbol{x} \geq 0\right\} .
$$

The discrete problem is now easily derived from problem (4.3) by the Galerkin method. It reads:

Find $\left(u_{1 h}, u_{2 h}, \sigma_{h}\right)$ in $\mathbb{X}_{h} \times \mathbb{X}_{h} \times \widetilde{\Lambda}_{h}^{*}$ such that

$$
\begin{gathered}
\forall\left(v_{1 h}, v_{2 h}\right) \in \mathbb{X}_{h} \times \mathbb{X}_{h}, \\
\sum_{i=1}^{2} \mu_{i} \int_{\omega}\left(\operatorname{grad} u_{i h}\right)(\boldsymbol{x}) \cdot\left(\operatorname{grad} v_{i h}\right)(\boldsymbol{x}) \mathrm{d} \boldsymbol{x} \\
\quad-\int_{\omega}\left(\operatorname{grad} \sigma_{h}\right)(\boldsymbol{x}) \cdot\left(\operatorname{grad}\left(v_{1 h}-v_{2 h}\right)\right)(\boldsymbol{x}) \mathrm{d} \boldsymbol{x}=\sum_{i=1}^{2}\left\langle f_{i}, v_{i h}\right\rangle, \\
\forall \varphi_{h} \in \widetilde{\Lambda}_{h}^{*}, \quad \int_{\omega}\left(\operatorname{grad}\left(\varphi_{h}-\sigma_{h}\right)\right)(\boldsymbol{x}) \cdot\left(\operatorname{grad}\left(u_{1 h}-u_{2 h}\right)\right)(\boldsymbol{x}) \mathrm{d} \boldsymbol{x} \geq 0 .
\end{gathered}
$$

The analysis of this problem relies on the properties of the forms $a(\cdot, \cdot)$ and $\tilde{b}(\cdot, \cdot)$. Indeed, the ellipticity property (3.6) is still valid on $\mathbb{X}_{h}$. We now check the inf-sup condition on $\tilde{b}(\cdot, \cdot)$, which relies on exactly the same arguments as for the continuous problem, see (4.5).

Lemma 5.1. The following inf-sup condition holds

$$
\forall \varphi_{h} \in \mathbb{X}_{h}, \quad \sup _{\boldsymbol{v}_{h} \in \mathbb{X}_{h}^{2}} \frac{\tilde{b}\left(\boldsymbol{v}_{h}, \varphi_{h}\right)}{\left|\boldsymbol{v}_{h}\right|_{H^{1}(\omega)^{2}}} \geq\left|\varphi_{h}\right|_{H^{1}(\omega)}
$$

Proof. When taking $\boldsymbol{v}_{h}=\left(0, \varphi_{h}\right)$, we have

$$
\tilde{b}\left(\boldsymbol{v}_{h}, \varphi_{h}\right)=\left|\varphi_{h}\right|_{H^{1}(\omega)}^{2} \quad \text { and } \quad\left|\boldsymbol{v}_{h}\right|_{H^{1}(\omega)^{2}}=\left|\varphi_{h}\right|_{H^{1}(\omega)},
$$

which gives the desired condition.

Moreover, the kernel $V_{h}$ of the form $\tilde{b}(\cdot, \cdot)$, i.e.,

$$
V_{h}=\left\{\boldsymbol{v}_{h}=\left(v_{1 h}, v_{2 h}\right) \in \mathbb{X}_{h} \times \mathbb{X}_{h} ; \forall \varphi_{h} \in \mathbb{X}_{h}, \tilde{b}\left(\boldsymbol{v}_{h}, \varphi_{h}\right)=0\right\}
$$


obviously satisfies

$$
V_{h}=\left\{\boldsymbol{v}_{h}=\left(v_{1 h}, v_{2 h}\right) \in \mathbb{X}_{h} \times \mathbb{X}_{h} ; v_{1 h}-v_{2 h}=0 \text { in } \omega\right\}=V \cap\left(\mathbb{X}_{h} \times \mathbb{X}_{h}\right) .
$$

Thus, the next statement is derived either from the same arguments as in Section 3 or by applying [18], Theorem 2.6 (see also [5], Thm. 2.1, or [13], Thm. 4.4).

Theorem 5.2. For any data $\left(f_{1}, f_{2}\right)$ in $H^{-1}(\omega) \times H^{-1}(\omega)$, problem (5.4) has a unique solution $\left(u_{1 h}, u_{2 h}, \sigma_{h}\right)$ in $\mathbb{X}_{h} \times \mathbb{X}_{h} \times \widetilde{\Lambda}_{h}^{*}$. Moreover, this solution satisfies

$$
\left\|u_{1 h}\right\|_{H^{1}(\omega)}+\left\|u_{2 h}\right\|_{H^{1}(\omega)}+\left\|\sigma_{h}\right\|_{H^{1}(\omega)} \leq c\left(\left\|f_{1}\right\|_{H^{-1}(\omega)}+\left\|f_{2}\right\|_{H^{-1}(\omega)}\right) .
$$

Let $\mathcal{K}_{h}^{*}$ denote the cone

$$
\mathcal{K}_{h}^{*}=\left\{\boldsymbol{v}_{h}=\left(v_{1 h}, v_{2 h}\right) \in \mathbb{X}_{h} \times \mathbb{X}_{h} ; \forall \varphi_{h} \in \widetilde{\Lambda}_{h}^{*}, \tilde{b}\left(\boldsymbol{v}_{h}, \varphi_{h}\right) \leq 0\right\} .
$$

Then, for any solution $\left(u_{1 h}, u_{2 h}, \sigma_{h}\right)$ of problem (5.4), the pair $\left(u_{1 h}, u_{2 h}\right)$ is the solution in $\mathcal{K}_{h}^{*}$ of the problem

$$
\forall\left(v_{1 h}, v_{2 h}\right) \in \mathcal{K}_{h}^{*}, \quad \sum_{i=1}^{2} \mu_{i} \int_{\omega}\left(\operatorname{grad} u_{i h}\right)(\boldsymbol{x}) \cdot\left(\operatorname{grad}\left(v_{i h}-u_{i h}\right)\right)(\boldsymbol{x}) \mathrm{d} \boldsymbol{x} \geq \sum_{i=1}^{2}\left\langle f_{i}, v_{i h}-u_{i h}\right\rangle .
$$

The consistency of our approach relies on the following discrete analogue of Lemma 4.4.

Lemma 5.3. The cone $\mathcal{K}_{h}^{*}$ defined in (5.9) coincides with the set

$$
\mathcal{K}_{h}=\left\{\left(v_{1 h}, v_{2 h}\right) \in \mathbb{X}_{h} \times \mathbb{X}_{h} ; v_{1 h}-v_{2 h} \geq 0 \text { in } \omega\right\} .
$$

Proof. It follows from the definition (5.3) of $\widetilde{\Lambda}_{h}^{*}$ that $\mathcal{K}_{h}$ is contained in $\mathcal{K}_{h}^{*}$. On the other hand, the following identity can be found in [10], Chapitre III, Section 5, for instance:

$$
\Lambda_{h}=\left\{v_{h} \in \mathbb{X}_{h} ; \forall \varphi_{h} \in \widetilde{\Lambda}_{h}^{*}, \int_{\omega}\left(\operatorname{grad} \varphi_{h}\right)(\boldsymbol{x}) \cdot\left(\operatorname{grad} v_{h}\right)(\boldsymbol{x}) \mathrm{d} \boldsymbol{x} \geq 0\right\} .
$$

Thus, if $\boldsymbol{v}_{h}$ belongs to $\mathcal{K}_{h}^{*}, v_{1 h}-v_{2 h}$ belongs to $\Lambda_{h}$, so that $\boldsymbol{v}_{h}$ belongs to $\mathcal{K}_{h}$.

To conclude, let us set: $\lambda_{h}=-\Delta \sigma_{h}$ in the sense of distributions. In analogy with Section 4, see (4.8), the action of $\lambda_{h}$ on a membrane with displacement $v$ can easily be recovered from the formula

$$
\forall v \in H_{0}^{1}(\omega), \quad\left\langle\lambda_{h}, v\right\rangle=\int_{\omega}\left(\operatorname{grad} \sigma_{h}\right)(\boldsymbol{x}) \cdot(\operatorname{grad} v)(\boldsymbol{x}) \mathrm{d} \boldsymbol{x} .
$$

When denoting by $\mathcal{E}_{h}$ the set of edges of triangles of $\mathcal{T}_{h}$ which are not contained in $\partial \omega$, this formula can equivalently be written as

$$
\forall v \in H_{0}^{1}(\omega), \quad\left\langle\lambda_{h}, v\right\rangle=\sum_{e \in \mathcal{E}_{h}} \int_{e}\left[\partial_{n} \sigma_{h}\right]_{e}(\tau) v(\tau) \mathrm{d} \tau,
$$

where $[\cdot]_{e}$ stands for the jump through $e$ with the appropriate sign. So, this action is easy to evaluate.

\section{A PRIORI ERROR ESTIMATES}

Proving a priori error estimates between the solutions $\left(u_{1}, u_{2}, \sigma\right)$ of problem $(4.3)$ and $\left(u_{1 h}, u_{2 h}, \sigma_{h}\right)$ of problem (5.4) relies on similar arguments as in [5], Theorem 2.2, or [18], Lemma 2.7, but is simpler since the first line in (4.3) is an equation. We begin with a lemma. 
Lemma 6.1. Assume that the data $\left(f_{1}, f_{2}\right)$ belong to $L^{2}(\omega) \times L^{2}(\omega)$. Then, the following error estimate holds between the solutions $\left(u_{1}, u_{2}, \sigma\right)$ of problem (4.3) and $\left(u_{1 h}, u_{2 h}, \sigma_{h}\right)$ of problem (5.4):

$$
\begin{aligned}
\left\|u_{1}-u_{1 h}\right\|_{H^{1}(\omega)}+\left\|u_{2}-u_{2 h}\right\|_{H^{1}(\omega)} \leq c \inf _{\left(v_{1 h}, v_{2 h}\right) \in \mathcal{K}_{h}^{*}}\left(\left\|u_{1}-v_{1 h}\right\|_{H^{1}(\omega)}+\left\|u_{2}-v_{2 h}\right\|_{H^{1}(\omega)}\right. \\
\left.+\sqrt{\kappa\left(f_{1}, f_{2}\right)}\left(\left\|u_{1}-v_{1 h}\right\|_{L^{2}(\omega)}+\left\|u_{2}-v_{2 h}\right\|_{L^{2}(\omega)}\right)^{\frac{1}{2}}\right) \\
+c^{\prime} \inf _{\chi_{h} \in \widetilde{\Lambda}_{h}^{*}}\left(\left\|\sigma-\chi_{h}\right\|_{H^{1}(\omega)}+\sqrt{\kappa\left(f_{1}, f_{2}\right)}\left\|\sigma-\chi_{h}\right\|_{L^{2}(\omega)}^{\frac{1}{2}}\right),
\end{aligned}
$$

where the constant $\kappa\left(f_{1}, f_{2}\right)$ is given by

$$
\kappa\left(f_{1}, f_{2}\right)=\left\|f_{1}\right\|_{L^{2}(\omega)}+\left\|f_{2}\right\|_{L^{2}(\omega)} .
$$

Proof. Starting from (5.10), we have for any $\left(v_{1 h}, v_{2 h}\right)$ in $\mathcal{K}_{h}^{*}$

$$
-\sum_{i=1}^{2} \mu_{i} \int_{\omega}\left(\operatorname{grad} u_{i h}\right)(\boldsymbol{x}) \cdot\left(\operatorname{grad}\left(v_{i h}-u_{i h}\right)\right)(\boldsymbol{x}) \mathrm{d} \boldsymbol{x} \leq-\sum_{i=1}^{2}\left\langle f_{i}, v_{i h}-u_{i h}\right\rangle .
$$

Thus, inserting the first line of (4.3) in this inequality (with each $v_{i}$ equal to $v_{i h}-u_{i h}$ ) and adding on both sides the appropriate integrals, we obtain

$$
\begin{aligned}
& \sum_{i=1}^{2} \mu_{i} \int_{\omega}\left(\operatorname{grad}\left(v_{i h}-u_{i h}\right)\right)(\boldsymbol{x}) \cdot\left(\operatorname{grad}\left(v_{i h}-u_{i h}\right)\right)(\boldsymbol{x}) \mathrm{d} \boldsymbol{x} \\
& \quad \leq \sum_{i=1}^{2} \mu_{i} \int_{\omega}\left(\operatorname{grad}\left(v_{i h}-u_{i}\right)\right)(\boldsymbol{x}) \cdot\left(\operatorname{grad}\left(v_{i h}-u_{i h}\right)\right)(\boldsymbol{x}) \mathrm{d} \boldsymbol{x}-\tilde{b}\left(\boldsymbol{v}_{h}-\boldsymbol{u}_{h}, \sigma\right)
\end{aligned}
$$

To evaluate the last term we observe that, for any $\chi_{h}$ in $\widetilde{\Lambda}_{h}^{*}$,

$$
-\tilde{b}\left(\boldsymbol{v}_{h}-\boldsymbol{u}_{h}, \sigma\right)=-\tilde{b}\left(\boldsymbol{v}_{h}-\boldsymbol{u}_{h}, \sigma-\chi_{h}\right)-\tilde{b}\left(\boldsymbol{v}_{h}-\boldsymbol{u}_{h}, \chi_{h}\right)
$$

whence, by using the fact that $\left(u_{1 h}, u_{2 h}\right)$ belongs to the cone $\mathcal{K}_{h}^{*}$ introduced in $(5.9)$,

$$
-\tilde{b}\left(\boldsymbol{v}_{h}-\boldsymbol{u}_{h}, \sigma\right) \leq-\tilde{b}\left(\boldsymbol{v}_{h}-\boldsymbol{u}_{h}, \sigma-\chi_{h}\right)-\tilde{b}\left(\boldsymbol{v}_{h}, \chi_{h}\right)
$$

This in turn leads to

$$
-\tilde{b}\left(\boldsymbol{v}_{h}-\boldsymbol{u}_{h}, \sigma\right) \leq-\tilde{b}\left(\boldsymbol{v}_{h}-\boldsymbol{u}_{h}, \sigma-\chi_{h}\right)+\tilde{b}\left(\boldsymbol{v}_{h}, \sigma-\chi_{h}\right)-\tilde{b}\left(\boldsymbol{v}_{h}, \sigma\right)
$$

It follows from the second line of problem (4.3) by taking successively $\varphi$ equal to zero, next $\varphi$ equal to $2 \sigma$, that $\tilde{b}(\boldsymbol{u}, \sigma)=0$. Thus, we derive

$$
-\tilde{b}\left(\boldsymbol{v}_{h}-\boldsymbol{u}_{h}, \sigma\right) \leq-\tilde{b}\left(\boldsymbol{v}_{h}-\boldsymbol{u}_{h}, \sigma-\chi_{h}\right)+\tilde{b}\left(\boldsymbol{v}_{h}, \sigma-\chi_{h}\right)+\tilde{b}\left(\boldsymbol{u}-\boldsymbol{v}_{h}, \sigma\right),
$$

whence finally

$$
-\tilde{b}\left(\boldsymbol{v}_{h}-\boldsymbol{u}_{h}, \sigma\right) \leq-\tilde{b}\left(\boldsymbol{v}_{h}-\boldsymbol{u}_{h}, \sigma-\chi_{h}\right)-\tilde{b}\left(\boldsymbol{u}-\boldsymbol{v}_{h}, \sigma-\chi_{h}\right)+\tilde{b}\left(\boldsymbol{u}, \sigma-\chi_{h}\right)+\tilde{b}\left(\boldsymbol{u}-\boldsymbol{v}_{h}, \sigma\right) .
$$


To bound the last two terms in this inequality, we observe, by integration by parts, that

$$
\begin{aligned}
& \left|\tilde{b}\left(\boldsymbol{u}, \sigma-\chi_{h}\right)\right| \leq\left(\left\|\Delta u_{1}\right\|_{L^{2}(\omega)}+\left\|\Delta u_{2}\right\|_{L^{2}(\omega)}\right)\left\|\sigma-\chi_{h}\right\|_{L^{2}(\omega)}, \\
& \left|\tilde{b}\left(\boldsymbol{u}-\boldsymbol{v}_{h}, \sigma\right)\right| \leq\left(\left\|u_{1}-v_{1 h}\right\|_{L^{2}(\omega)}+\left\|u_{2}-v_{2 h}\right\|_{L^{2}(\omega)}\right)\|\Delta \sigma\|_{L^{2}(\omega)} .
\end{aligned}
$$

Proposition 3.6 and problem (3.1) also yield that

$$
\|\Delta \sigma\|_{L^{2}(\omega)}+\left\|\Delta u_{1}\right\|_{L^{2}(\omega)}+\left\|\Delta u_{2}\right\|_{L^{2}(\omega)} \leq c \kappa\left(f_{1}, f_{2}\right) .
$$

The desired result follows from (6.3) to (6.6) combined with a triangle inequality.

The next lemma requires the orthogonal projection operator $\Pi_{h}$ from $H_{0}^{1}(\omega)$ onto $\mathbb{X}_{h}$ : For any $\varphi$ in $H_{0}^{1}(\omega)$, $\Pi_{h} \varphi$ belongs to $\mathbb{X}_{h}$ and satisfies

$$
\forall \psi_{h} \in \mathbb{X}_{h}, \quad \int_{\omega}\left(\operatorname{grad}\left(\varphi-\Pi_{h} \varphi\right)\right)(\boldsymbol{x}) \cdot\left(\operatorname{grad} \psi_{h}\right)(\boldsymbol{x}) \mathrm{d} \boldsymbol{x}=0 .
$$

Lemma 6.2. Assume that the domain $\omega$ is convex. For any function $\chi$ in $\widetilde{\Lambda}^{*} \cap H^{s+1}(\omega), 0 \leq s \leq 1$, the function $\Pi_{h} \chi$ belongs to $\widetilde{\Lambda}_{h}^{*}$ and satisfies

$$
\left\|\chi-\Pi_{h} \chi\right\|_{H^{1}(\omega)} \leq c h^{s}\|\chi\|_{H^{s+1}(\omega)}, \quad\left\|\chi-\Pi_{h} \chi\right\|_{L^{2}(\omega)} \leq c h^{s+1}\|\chi\|_{H^{s+1}(\omega)} .
$$

Proof. We have, for any $v_{h}$ in $\Lambda_{h}$,

$$
\int_{\omega}\left(\operatorname{grad} \Pi_{h} \chi\right)(\boldsymbol{x}) \cdot\left(\operatorname{grad} v_{h}\right)(\boldsymbol{x}) \mathrm{d} \boldsymbol{x}=\int_{\omega}(\operatorname{grad} \chi)(\boldsymbol{x}) \cdot\left(\operatorname{grad} v_{h}\right)(\boldsymbol{x}) \mathrm{d} \boldsymbol{x} .
$$

Since $\Lambda_{h}$ is contained in $\Lambda$, this yields

$$
\int_{\omega}\left(\operatorname{grad} \Pi_{h} \chi\right)(\boldsymbol{x}) \cdot\left(\operatorname{grad} v_{h}\right)(\boldsymbol{x}) \mathrm{d} \boldsymbol{x} \geq 0,
$$

so that $\Pi_{h} \chi$ belongs to $\widetilde{\Lambda}_{h}^{*}$. The approximation error estimates (6.8) can be derived from [3], Théorème IX.2.3 and Corollaire IX.2.4, for instance and an interpolation argument.

The next lemma requires the Lagrange interpolation operator $\mathcal{I}_{h}$ at all vertices of elements $K$ of $\mathcal{T}_{h}$ which are inside $\omega$ with values in $\mathbb{X}_{h}$.

Lemma 6.3. For any function $\boldsymbol{v}=\left(v_{1}, v_{2}\right)$ in $\mathcal{K} \cap H^{s+1}(\omega)^{2}, 0<s \leq 1$, the function $\mathcal{I}_{h} \boldsymbol{v}$ belongs to $\mathcal{K}_{h}^{*}$ and satisfies

$$
\left\|\boldsymbol{v}-\mathcal{I}_{h} \boldsymbol{v}\right\|_{H^{1}(\omega)^{2}} \leq c h^{s}\|\boldsymbol{v}\|_{H^{s+1}(\omega)^{2}}, \quad\left\|\boldsymbol{v}-\mathcal{I}_{h} \boldsymbol{v}\right\|_{L^{2}(\omega)^{2}} \leq c h^{s+1}\|\boldsymbol{v}\|_{H^{s+1}(\omega)^{2}} .
$$

Proof. Owing to the definitions (3.10) of $\mathcal{K}$ and (5.2) of $\Lambda_{h}$, since $\mathcal{I}_{h}\left(v_{1}-v_{2}\right)$ is affine on each $K$ in $\mathcal{T}_{h}$ and nonnegative at each vertex of $K$, then $\mathcal{I}_{h}\left(v_{1}-v_{2}\right)$ belongs to $\Lambda_{h}$. Thus, Lemma 5.3 implies that the pair $\left(\mathcal{I}_{h} v_{1}, \mathcal{I}_{h} v_{2}\right)$ belongs to the cone $\mathcal{K}_{h}^{*}$ introduced in (5.9). Its approximation properties (6.9) are established in [8], Theorem 17.1, or [3], Théorèmes IX.1.5 and IX.1.6, for instance.

By inserting the results of Lemmas 6.2 and 6.3 in (6.1) and using the regularity results of Proposition 4.3, we obtain the first error estimate.

Theorem 6.4. Assume that the domain $\omega$ is convex and that the data $\left(f_{1}, f_{2}\right)$ belong to $L^{2}(\omega) \times L^{2}(\omega)$. Then, the following a priori error estimate holds between the solutions $\left(u_{1}, u_{2}, \sigma\right)$ of problem (4.3) and $\left(u_{1 h}, u_{2 h}, \sigma_{h}\right)$ of problem (5.4)

$$
\left\|u_{1}-u_{1 h}\right\|_{H^{1}(\omega)}+\left\|u_{2}-u_{2 h}\right\|_{H^{1}(\omega)} \leq \operatorname{ch} \kappa\left(f_{1}, f_{2}\right),
$$

where the constant $\kappa\left(f_{1}, f_{2}\right)$ is introduced in (6.2). 
Estimate (6.10) is fully optimal, since the discretization that we propose is of order 1. But, as standard for variational inequalities (see for instance [8], Thm. 23.1), it would not be optimal if higher discretizations, relying on piecewise polynomial functions with degree $\geq 2$, were considered.

Remark 6.5. When $\omega$ is not convex, standard arguments and Proposition 4.3 yield estimate (6.10), with $h$ replaced by $h^{s}$ for all $s<\frac{\pi}{\alpha}$, where $\alpha$ denotes the largest angle internal to $\omega$. Moreover, estimate (6.10) still holds when the mesh is exponentially refined near the re-entrant corners, as first suggested in [17].

To go further, we now estimate the distance between $\sigma$ and $\sigma_{h}$. The following result, which can also be found in [5], Theorem 2.3, is easily derived by subtracting the first line of (5.4) from the first line in (4.3) (with $\boldsymbol{v}=\boldsymbol{v}_{h}$ in $\mathbb{X}_{h}^{2}$ ) and using the inf-sup condition (5.5).

Lemma 6.6. The following error estimate holds between the solutions $\left(u_{1}, u_{2}, \sigma\right)$ of problem (4.3) and $\left(u_{1 h}, u_{2 h}, \sigma_{h}\right)$ of problem $(5.4)$ :

$$
\left\|\sigma-\sigma_{h}\right\|_{H^{1}(\omega)} \leq c\left(\left\|u_{1}-u_{1 h}\right\|_{H^{1}(\omega)}+\left\|u_{2}-u_{2 h}\right\|_{H^{1}(\omega)}+\inf _{\chi_{h} \in \mathbb{X}_{h}}\left\|\sigma-\chi_{h}\right\|_{H^{1}(\omega)}\right) .
$$

This yields the final result.

Corollary 6.7. Assume that the domain $\omega$ is convex and that the data $\left(f_{1}, f_{2}\right)$ belong to $L^{2}(\omega) \times L^{2}(\omega)$. Then, the following a priori error estimate holds between the solutions $\left(u_{1}, u_{2}, \sigma\right)$ of problem $(4.3)$ and $\left(u_{1 h}, u_{2 h}, \sigma_{h}\right)$ of problem (5.4)

$$
\left\|\sigma-\sigma_{h}\right\|_{H^{1}(\omega)} \leq \operatorname{ch} \kappa\left(f_{1}, f_{2}\right)
$$

where the constant $\kappa\left(f_{1}, f_{2}\right)$ is introduced in (6.2).

A more physical version of Corollary 6.7 concerns the error between the action $\lambda$ and the discrete action $\lambda_{h}$. Indeed, we easily derive from (4.8) and (5.12) that, if the assumptions of Corollary 6.7 hold,

$$
\left\|\lambda-\lambda_{h}\right\|_{H^{-1}(\omega)} \leq \operatorname{ch\kappa }\left(f_{1}, f_{2}\right) .
$$

This can equivalently be stated as follows: For any membrane with displacement $v$ in $H_{0}^{1}(\omega)$,

$$
\frac{\left|\langle\lambda, v\rangle-\left\langle\lambda_{h}, v\right\rangle\right|}{\|v\|_{H^{1}(\omega)}} \leq \operatorname{ch\kappa }\left(f_{1}, f_{2}\right) .
$$

\section{A POSTERIORI ERROR ESTIMATES}

In order to define the error indicators, we need some further notation. We recall from Section 5 that $\mathcal{E}_{h}$ is the set of edges of elements of $\mathcal{T}_{h}$ which are not contained in $\partial \Omega$.

- For each $K$ in $\mathcal{T}_{h}, \mathcal{E}_{K}$ is the set of edges of $K$ which belong to $\mathcal{E}_{h}$.

- For each $e$ in $\mathcal{E}_{h}$, we denote by $h_{e}$ the length of $e$ and by $[\cdot]_{e}$ the jump through $e$ : More precisely, if $\boldsymbol{n}$ is a unit normal vector to $e$ directed from a triangle $K$ to another triangle $K^{\prime}$, for any smooth enough vector field $\boldsymbol{w},[\boldsymbol{w} \cdot \boldsymbol{n}]_{e}$ denotes the quantity $\left(\boldsymbol{w}_{\mid K^{\prime}}-\boldsymbol{w}_{\mid K}\right) \cdot \boldsymbol{n}$.

- For any smooth enough function $v$ on an edge $e$, the function $v^{+}$is the positive part of $v$, defined as $\max \{v, 0\}$.

We introduce an approximation $\left(f_{1 h}, f_{2 h}\right)$ of the data $\left(f_{1}, f_{2}\right)$, which is constant on each element $K$ of $\mathcal{T}_{h}$. Following the ideas in [14], Section 4, we define two types of error indicators: For any triangle $K$ in $\mathcal{T}_{h}$ and any $e$ in $\mathcal{E}_{h}$,

- $\eta_{K}$ is the standard residual-type indicator for elliptic equations

$$
\begin{aligned}
\eta_{K}=h_{K}(\operatorname{meas} K)^{\frac{1}{2}}\left(\left|f_{1 h}\right|_{K} \mid\right. & \left.+\left|f_{2 h}\right|_{K} \mid\right) \\
& +\sum_{e \in \mathcal{E}_{K}} h_{e}\left(\left|\left[\partial_{n}\left(\mu_{1} u_{1 h}-\sigma_{h}\right)\right]_{e}\right|+\left|\left[\partial_{n}\left(\mu_{2} u_{2 h}+\sigma_{h}\right)\right]_{e}\right|\right)
\end{aligned}
$$


- $\eta_{e}$ comes from the lack of positivity of $\lambda_{h}=-\Delta \sigma_{h}$ (in a weak sense)

$$
\eta_{e}=\left(h_{e}\left[\partial_{n} \sigma_{h}\right]_{e}^{+}\right)^{\frac{1}{2}} .
$$

All these indicators are easy to compute once the discrete solution is known, since they only involve constant quantities.

Let us write the residual equation associated with the first line of problem (4.3). It reads, for any $\boldsymbol{v}$ in $H_{0}^{1}(\Omega)^{2}$ and $\boldsymbol{v}_{h}$ in $\mathbb{X}_{h}^{2}$,

$$
\begin{aligned}
\sum_{i=1}^{2} \mu_{i} \int_{\omega}\left(\operatorname{grad}\left(u_{i}-u_{i h}\right)\right)(\boldsymbol{x}) \cdot\left(\operatorname{grad} v_{i}\right)(\boldsymbol{x}) \mathrm{d} \boldsymbol{x}+\widetilde{b}\left(\boldsymbol{v}, \sigma-\sigma_{h}\right) & \\
= & \sum_{i=1}^{2}\left(\left\langle f_{i}-f_{i h}, v_{i}-v_{i h}\right\rangle+\left\langle R_{i}, v_{i}-v_{i h}\right\rangle\right),
\end{aligned}
$$

where the residuals $R_{i}$ are given after integration by parts on each $K$ by

$$
\left\langle R_{i}, v_{i}\right\rangle=\sum_{K \in \mathcal{T}_{h}}\left(\int_{K} f_{i h}(\boldsymbol{x}) v_{i}(\boldsymbol{x}) \mathrm{d} \boldsymbol{x}-\int_{\partial K}\left(\partial_{n}\left(\mu_{i} u_{i h}+(-1)^{i} \sigma_{h}\right)(\tau) v_{i}(\tau) \mathrm{d} \tau\right) .\right.
$$

Next, we introduce a Clément-type regularization operator $\mathcal{C}_{h}$ with values in $\mathbb{X}_{h}$, which satisfies, for each $K$ in $\mathcal{T}_{h}$ and each $e$ in $\mathcal{E}_{h}$ and for any function $v$ in $H_{0}^{1}(\omega)$ (see [9] and [3], Thm. IX.3.7 and Cor. IX.3.9, for instance)

$$
\left\|v-\mathcal{C}_{h} v\right\|_{L^{2}(K)} \leq c h_{K}\|v\|_{H^{1}\left(\Delta_{K}\right)}, \quad\left\|v-\mathcal{C}_{h} v\right\|_{L^{2}(e)} \leq c^{\prime} h_{e}^{\frac{1}{2}}\|v\|_{H^{1}\left(\Delta_{e}\right)},
$$

where $\Delta_{K}$ and $\Delta_{e}$ stand for the union of the elements of $\mathcal{T}_{h}$ that intersect $K$ and $e$, respectively. Thus, when taking $v_{i h}$ equal to $\mathcal{C}_{h} v_{i}$, it is readily checked that

$$
\sum_{i=1}^{2}\left(\left\langle f_{i}-f_{i h}, v_{i}-v_{i h}\right\rangle+\left\langle R_{i}, v_{i}-v_{i h}\right\rangle\right) \leq c\left(\sum_{K \in \mathcal{T}_{h}}\left(h_{K}^{2} \sum_{i=1}^{2}\left\|f_{i}-f_{i h}\right\|_{L^{2}(K)}^{2}+\left(\eta_{K}\right)^{2}\right)\right)^{\frac{1}{2}}\|\boldsymbol{v}\|_{H^{1}(\omega)^{2}} .
$$

A further argument is needed to evaluate the quantity $\tilde{b}\left(\boldsymbol{u}-\boldsymbol{u}_{h}, \sigma-\sigma_{h}\right)$.

Lemma 7.1. The following estimate holds

$$
-\tilde{b}\left(\boldsymbol{u}-\boldsymbol{u}_{h}, \sigma-\sigma_{h}\right) \leq \kappa\left(f_{1}, f_{2}\right) \sum_{e \in \mathcal{E}_{h}} \eta_{e}^{2},
$$

where the constant $\kappa\left(f_{1}, f_{2}\right)$ is introduced in (6.2).

Proof. We have

$$
-\tilde{b}\left(\boldsymbol{u}-\boldsymbol{u}_{h}, \sigma-\sigma_{h}\right)=-\tilde{b}(\boldsymbol{u}, \sigma)+\tilde{b}\left(\boldsymbol{u}, \sigma_{h}\right)+\tilde{b}\left(\boldsymbol{u}_{h}, \sigma\right)-\tilde{b}\left(\boldsymbol{u}_{h}, \sigma_{h}\right) .
$$

It follows from the second line of (4.3) by taking $\varphi$ successively equal to 0 and $2 \sigma$ that $\tilde{b}(\boldsymbol{u}, \sigma)=0$. Exactly the same arguments applied to the second line of (5.4) yield that $\tilde{b}\left(\boldsymbol{u}_{h}, \sigma_{h}\right)=0$. On the other hand, it follows from Lemma 5.3 that $u_{1 h}-u_{2 h}$ belongs to $\Lambda$ and $\sigma$ belongs to $\widetilde{\Lambda}^{*} ; \tilde{b}\left(\boldsymbol{u}_{h}, \sigma\right)$ is thus nonpositive. So we derive

$$
-\tilde{b}\left(\boldsymbol{u}-\boldsymbol{u}_{h}, \sigma-\sigma_{h}\right) \leq \tilde{b}\left(\boldsymbol{u}, \sigma_{h}\right) .
$$

To evaluate this last term, we observe by integration by parts that

$$
\tilde{b}\left(\boldsymbol{u}, \sigma_{h}\right)=\sum_{e \in \mathcal{E}_{h}} \int_{e}\left[\partial_{n} \sigma_{h}\right]_{e}\left(u_{1}-u_{2}\right)(\tau) \mathrm{d} \tau,
$$


whence

$$
\tilde{b}\left(\boldsymbol{u}, \sigma_{h}\right) \leq \sum_{e \in \mathcal{E}_{h}}\left[\partial_{n} \sigma_{h}\right]_{e}^{+} \int_{e}\left(u_{1}-u_{2}\right)(\tau) \mathrm{d} \tau .
$$

On the other hand, Proposition 4.3 combined with the Sobolev imbedding of $H^{s+1}(\omega), s>0$, into the space of continuous functions on $\bar{\omega}$ yields that

$$
\sup _{\boldsymbol{x} \in \bar{\omega}}\left|\left(u_{1}-u_{2}\right)(\boldsymbol{x})\right| \leq \kappa\left(f_{1}, f_{2}\right) .
$$

We obtain the desired estimate by combining (7.7) to (7.9).

The a posteriori error estimate for the error is now easily derived by taking $\boldsymbol{v}$ equal to $\boldsymbol{u}-\boldsymbol{u}_{h}$ in $(7.3)$ and using (7.5) and (7.6).

Theorem 7.2. Assume that the data $\left(f_{1}, f_{2}\right)$ belong to $L^{2}(\omega) \times L^{2}(\omega)$. Then, the following a posteriori error estimate holds between the solutions $\left(u_{1}, u_{2}, \sigma\right)$ of problem (4.3) and $\left(u_{1 h}, u_{2 h}, \sigma_{h}\right)$ of problem (5.4)

$$
\begin{aligned}
& \left\|u_{1}-u_{1 h}\right\|_{H^{1}(\omega)}+\left\|u_{2}-u_{2 h}\right\|_{H^{1}(\omega)} \\
& \quad \leq c\left(\sum_{K \in \mathcal{T}_{h}}\left(h_{K}^{2} \sum_{i=1}^{2}\left\|f_{i}-f_{i h}\right\|_{L^{2}(K)}^{2}+\left(\eta_{K}\right)^{2}\right)\right)^{\frac{1}{2}}+\sqrt{\kappa\left(f_{1}, f_{2}\right)}\left(\sum_{e \in \mathcal{E}_{h}}\left(\eta_{e}\right)^{2}\right)^{\frac{1}{2}},
\end{aligned}
$$

where the constant $\kappa\left(f_{1}, f_{2}\right)$ is introduced in (6.2).

On the other hand, applying the inf-sup condition (4.5) in (7.3) yields

$$
\begin{aligned}
\left|\sigma-\sigma_{h}\right|_{H^{1}(\omega)} \leq \sum_{i=1}^{2} \mu_{i}\left|u_{i}-u_{i h}\right|_{H^{1}(\omega)} & \\
& +\sup _{\boldsymbol{v} \in H_{0}^{1}(\omega)^{2}} \frac{\sum_{i=1}^{2}\left(\left\langle f_{i}-f_{i h}, v_{i}-\mathcal{C}_{h} v_{i}\right\rangle+\left\langle R_{i}, v_{i}-\mathcal{C}_{h} v_{i}\right\rangle\right)}{|\boldsymbol{v}|_{H^{1}}(\omega)^{2}} .
\end{aligned}
$$

So, the estimate for $\left\|\sigma-\sigma_{h}\right\|_{H^{1}(\omega)}$ is easily derived from (7.6) and Theorem 7.2.

Corollary 7.3. Assume that the data $\left(f_{1}, f_{2}\right)$ belong to $L^{2}(\omega) \times L^{2}(\omega)$. Then, the following a posteriori error estimate holds between the solutions $\left(u_{1}, u_{2}, \sigma\right)$ of problem (4.3) and $\left(u_{1 h}, u_{2 h}, \sigma_{h}\right)$ of problem (5.4)

$$
\left\|\sigma-\sigma_{h}\right\|_{H^{1}(\omega)} \leq c\left(\sum_{K \in \mathcal{T}_{h}}\left(h_{K}^{2} \sum_{i=1}^{2}\left\|f_{i}-f_{i h}\right\|_{L^{2}(K)}^{2}+\left(\eta_{K}\right)^{2}\right)\right)^{\frac{1}{2}}+c^{\prime} \sqrt{\kappa\left(f_{1}, f_{2}\right)}\left(\sum_{e \in \mathcal{E}_{h}}\left(\eta_{e}\right)^{2}\right)^{\frac{1}{2}}
$$

where the constant $\kappa\left(f_{1}, f_{2}\right)$ is introduced in (6.2).

We now intend to prove an upper bound for each indicator $\eta_{K}$ and $\eta_{e}$. As standard, the bound for $\eta_{K}$ follows from appropriate choices of $\boldsymbol{v}$ in (7.3) and inverse inequalities (see [19], Sect. 3.1). So we only give an abridged proof of the next statement.

Theorem 7.4. The following bound holds for each indicator $\eta_{K}, K \in \mathcal{T}_{h}$, defined in (7.1)

$$
\begin{aligned}
\eta_{K} \leq c\left(\left\|u_{1}-u_{1 h}\right\|_{H^{1}\left(\omega_{K}\right)}+\| u_{2}\right. & -u_{2 h}\left\|_{H^{1}\left(\omega_{K}\right)}+\right\| \sigma-\sigma_{h} \|_{H^{1}\left(\omega_{K}\right)} \\
& \left.+h_{K}\left\|f_{1}-f_{1 h}\right\|_{L^{2}\left(\omega_{K}\right)}+h_{K}\left\|f_{2}-f_{2 h}\right\|_{L^{2}\left(\omega_{K}\right)}\right),
\end{aligned}
$$

where $\omega_{K}$ denotes the union of elements of $\mathcal{T}_{h}$ that share at least an edge with $K$. 
Proof. We bound successively the four terms in $\eta_{K}$.

(1) We set

$$
v_{i K}= \begin{cases}f_{i h} \psi_{K} & \text { on } K, \\ 0 & \text { on } \omega \backslash K,\end{cases}
$$

where $\psi_{K}$ denotes the bubble function on $K$ (equal to the product of the barycentric coordinates associated with the vertices of $K)$. Thus, taking $\boldsymbol{v}=\left(v_{1 K}, 0\right)$ and $\boldsymbol{v}_{h}=\mathbf{0}$ in (7.3) gives

$$
\begin{aligned}
\left\|f_{1 h} \psi_{K}^{\frac{1}{2}}\right\|_{L^{2}(K)}^{2} \leq\left(\mu_{1}\left|u_{1}-u_{1 h}\right|_{H^{1}\left(\omega_{K}\right)}+\left|\sigma-\sigma_{h}\right|_{H^{1}\left(\omega_{K}\right)}\right)\left|v_{1 K}\right|_{H^{1}(K)} \\
+\left\|f_{1}-f_{1 h}\right\|_{L^{2}(K)}\left\|v_{1 K}\right\|_{L^{2}(K)} .
\end{aligned}
$$

Thus, the bound for the first term is derived from appropriate inverse inequalities [19], Lemma 3.3, when multiplying this inequality by $h_{K}$.

(2) When taking $\boldsymbol{v}=\left(0, v_{2 K}\right)$, the same arguments lead to the bound for the second term.

(3) For each edge $e$ in $\mathcal{E}_{K}$, denoting by $K^{\prime}$ the other triangle that contains $e$, we now set

$$
v_{i e}= \begin{cases}l_{e, \kappa}\left(\left[\partial_{n}\left(\mu_{i} u_{i h}+(-1)^{i} \sigma_{h}\right)\right]_{e} \psi_{e}\right) & \text { on } \kappa \in\left\{K, K^{\prime}\right\}, \\ 0 & \text { on } \omega \backslash\left(K \cup K^{\prime}\right),\end{cases}
$$

where $\psi_{e}$ denotes the bubble function on $e$ and $l_{e, \kappa}$ is a lifting operator from functions on $e$ vanishing at the endpoints of $e$ into functions on $\kappa$ vanishing on $\partial \kappa \backslash e$ constructed from a fixed lifting operator on the reference triangle. Taking now $\boldsymbol{v}=\left(v_{1 e}, 0\right)$ and $\boldsymbol{v}_{h}=\mathbf{0}$ in (7.3) and using standard arguments and the bound for the first term in $\eta_{K}$ yield the desired estimate for the third term.

(4) The same arguments with now $\boldsymbol{v}=\left(0, v_{2 e}\right)$ leads to the estimate for the fourth term.

Theorem 7.5. Assume that the data $\left(f_{1}, f_{2}\right)$ belong to $L^{2}(\omega) \times L^{2}(\omega)$. The following bound holds for each indicator $\eta_{e}, e \in \mathcal{E}_{h}$, defined in (7.2), when not zero,

$$
\eta_{e} \leq c\left\|\sigma-\sigma_{h}\right\|_{H^{1}\left(\omega_{e}\right)}^{\frac{1}{2}},
$$

where $\omega_{e}$ denotes the union of the two elements of $\mathcal{T}_{h}$ that share $e$.

Proof. When $\eta_{e}$ is not zero, using once more an inverse inequality, we have

$$
\eta_{e}^{2}=\int_{e}\left[\partial_{n} \sigma_{h}\right]_{e}^{+} \mathrm{d} \tau \leq c \int_{e}\left[\partial_{n} \sigma_{h}\right]_{e} \psi_{e}(\tau) \mathrm{d} \tau \leq-c \int_{e}\left[\partial_{n}\left(\sigma-\sigma_{h}\right)\right]_{e}(\tau) \psi_{e}(\tau) \mathrm{d} \tau
$$

Thus, denoting by $K$ and $K^{\prime}$ the two triangles that share $e$, we obtain (here, $\boldsymbol{n}_{\kappa}$ denotes the unit outward normal vector to $\kappa$ )

$$
\begin{aligned}
& \eta_{e}^{2} \leq c \sum_{\kappa \in\left\{K, K^{\prime}\right\}} \int_{\partial \kappa} \partial_{n_{\kappa}}\left(\sigma-\sigma_{h}\right)(\tau) \psi_{e}(\tau) \mathrm{d} \tau \\
& \leq c \sum_{\kappa \in\left\{K, K^{\prime}\right\}}\left(\int_{\kappa}(\Delta \sigma)(\boldsymbol{x}) \psi_{e}(\boldsymbol{x}) \mathrm{d} \boldsymbol{x}+\int_{\kappa}\left(\operatorname{grad}\left(\sigma-\sigma_{h}\right)\right)(\boldsymbol{x}) \cdot\left(\operatorname{grad} \psi_{e}\right)(\boldsymbol{x}) \mathrm{d} \boldsymbol{x}\right) .
\end{aligned}
$$


Since $\Delta \sigma$ is $\leq 0$, we conclude by using Cauchy-Schwarz inequalities and noting that

$$
\left\|\psi_{e}\right\|_{L^{2}(\kappa)} \leq c h_{e}, \quad\left|\psi_{e}\right|_{H^{1}(\kappa)} \leq c,
$$

both inequalities being easily derived by switching to the reference triangle.

Estimates (7.10) and (7.12) are optimal. Estimate (7.13) is also optimal but estimate (7.15) is not because of the power $1 / 2$ on the norm $\left\|\sigma-\sigma_{h}\right\|_{H^{1}\left(\omega_{e}\right)}$. However the same lack of optimality already appears in [14], Section 4, for a similar problem, see also [6], Remark 4.7. Nevertheless, since these last estimates are fully local, it can be thought that the $\eta_{K}$ and $\eta_{e}$ provide a good representation of the local error and hence are an efficient tool for mesh adaptivity.

\section{Conclusions}

For the discretization that we propose, the a priori error estimates are fully optimal and the a posteriori error estimates seem the best as possible for variational inequalities. So, we think that this method leads to an efficient discretization of the contact problem.

In any case, another discretization of this model is under our consideration, where the initial unknown $\lambda$ is directly discretized via a finite volume scheme, which can be equivalently reduced to a Galerkin approximation of problem (3.11). In particular, it seems that we can here obtain optimal a posteriori error estimates. We intend to perform an analysis of this approach in a forthcoming paper and also to make numerical experiments in order to compare the two methods.

The model that we have studied in this paper is a first draft for the contact between several thin structures such as plates or shells. Indeed, in this last situation, the Laplace equations are replaced by more complex second order equations where the coefficients depend on the geometry of the structures. However, the equations modeling the contact rely on the same principles as for membranes. So, we think that a similar discretization could be appropriate for handling these new models.

\section{REFERENCES}

[1] M. Ainsworth, J.T. Oden and C.Y. Lee, Local a posteriori error estimators for variational inequalities. Numer. Methods Partial Differential Equations 9 (1993) 23-33.

[2] F. Ali Mehmeti and S. Nicaise, Nonlinear interaction problems. Nonlinear Anal. Theory Methods Appl. 20 (1993) $27-61$.

[3] C. Bernardi, Y. Maday and F. Rapetti, Discrétisations variationnelles de problèmes aux limites elliptiques, Collection Mathématiques \& Applications 45. Springer-Verlag (2004).

[4] H. Brezis and G. Stampacchia, Sur la régularité de la solution d'inéquations elliptiques. Bull. Soc. Math. France 96 (1968) $153-180$.

[5] F. Brezzi, W.W. Hager and P.-A. Raviart, Error estimates for the finite element solution of variational inequalities, II. Mixed methods. Numer. Math. 31 (1978-1979) 1-16.

[6] Z. Chen and R.H. Nochetto, Residual type a posteriori error estimates for elliptic obstacle problems. Numer. Math. 84 (2000) $527-548$.

[7] P.G. Ciarlet, The Finite Element Method for Elliptic Problems. North Holland, Amsterdam, New York, Oxford (1978).

[8] P.G. Ciarlet, Basic error estimates for elliptic problems, in Handbook of Numerical Analysis, Vol. II, P.G. Ciarlet and J.-L. Lions Eds., North-Holland, Amsterdam (1991) 17-351.

[9] P. Clément, Approximation by finite element functions using local regularization. RAIRO Anal. Numér. 9 R2 (1975) $77-84$.

[10] I. Ekeland and R. Temam, Analyse convexe et problèmes variationnels. Dunod \& Gauthier-Villars (1974).

[11] V. Girault and P.-A. Raviart, Finite Element Methods for Navier-Stokes Equations, Theory and Algorithms. Springer-Verlag (1986).

[12] P. Grisvard, Elliptic Problems in Nonsmooth Domains. Pitman (1985).

[13] J. Haslinger, I. Hlaváček and J. Nečas, Numerical methods for unilateral problems in solid mechanics, in Handbook of Numerical Analysis, Vol. IV, P.G. Ciarlet and J.-L. Lions Eds., North-Holland, Amsterdam (1996) 313-485.

[14] P. Hild and S. Nicaise, Residual a posteriori error estimators for contact problems in elasticity. ESAIM: M2AN 41 (2007) 897-923.

[15] J.-L. Lions and G. Stampacchia, Variational inequalities. Comm. Pure Appl. Math. 20 (1967) 493-519.

[16] R.H. Nochetto, K.G. Siebert and A. Veeser, Pointwise a posteriori error control for elliptic obstacle problems. Numer. Math. 95 (2003) 163-195. 
[17] G. Raugel, Résolution numérique par une méthode d'éléments finis du problème de Dirichlet pour le laplacien dans un polygone. C. R. Acad. Sci. Paris Sér. A-B 286 (1978) A791-A794.

[18] L. Slimane, A. Bendali and P. Laborde, Mixed formulations for a class of variational inequalities. ESAIM: M2AN 38 (2004) $177-201$.

[19] R. Verfürth, A Review of A Posteriori Error Estimation and Adaptive Mesh-Refinement Techniques. Wiley \& Teubner (1996).

[20] B.I. Wohlmuth, An a posteriori error estimator for two body contact problems on non-matching meshes. J. Sci. Computing 33 (2007) 25-45. 